\title{
The Diagnostic Value of Serum C-Reactive Protein for Identifying Pneumonia in Hospitalized Patients with Acute Respiratory Symptoms
}

\author{
Agustín Ruiz-González, Laia Utrillo, Silvia Bielsa, Miquel Falguera, and José M. Porcel \\ Department of Internal Medicine, Arnau de Vilanova University Hospital, Biomedical Research Institute of \\ Lleida Foundation Dr. Pifarré (IRBLleida), 25198 Lleida, Spain
}

Correspondence should be addressed to Agustín Ruiz-González; agustinruiz@saludalia.com

Received 10 May 2016; Accepted 25 July 2016

Academic Editor: Mark Duncan

Copyright (C) 2016 Agustín Ruiz-González et al. This is an open access article distributed under the Creative Commons Attribution License, which permits unrestricted use, distribution, and reproduction in any medium, provided the original work is properly cited.

Background. The clinical diagnosis of pneumonia is sometimes difficult since chest radiographs are often indeterminate. In this study, we aimed to assess whether serum C-reactive protein (CRP) could assist in identifying patients with pneumonia. Methods. For one winter, all consecutive patients with acute respiratory symptoms admitted to the emergency ward of a single center were prospectively enrolled. In addition to chest radiographs, basic laboratory tests, and microbiology, serum levels of CRP were measured at entry. Results. A total of 923 (62.3\%) of 1473 patients hospitalized for acute respiratory symptoms were included. Subjects with a final diagnosis of pneumonia had higher serum CRP levels (median $187 \mathrm{mg} / \mathrm{L}$ ) than those with exacerbations of chronic obstructive pulmonary disease $(63 \mathrm{mg} / \mathrm{L})$ or acute bronchitis $(54 \mathrm{mg} / \mathrm{L}, p<0.01)$. CRP was accurate in identifying pneumonia (area under the curve $0.84,95 \%$ CI 0.82-0.87). The multilevel likelihood ratio (LR) for intervals of CRP provided useful information on the posttest probability of having pneumonia. CRP intervals above $200 \mathrm{mg} / \mathrm{L}$ were associated with LR+ $>5$, for which pneumonia is likely, whereas CRP intervals below $75 \mathrm{mg} / \mathrm{L}$ were associated with $\mathrm{LR}<0.2$, for which pneumonia is unlikely. Conclusion. Serum CRP may be a useful addition for diagnosing pneumonia in hospitalized patients with acute respiratory symptoms.

\section{Introduction}

Pneumonia is a leading cause of hospitalization and death in developed countries [1]. However, the discrimination of pneumonia from other lower respiratory tract infections (LRTI), where antibiotics are not required, is sometimes challenging, particularly in its early stages. In elderly patients, the clinical presentation is often nonspecific and interpreting chest radiographs can be difficult in patients with severe or previous pulmonary disease $[2,3]$.

Recent guidelines and review studies have suggested that serum C-reactive protein (CRP) may be helpful in distinguishing pneumonia from other acute respiratory illnesses $[4,5]$. Nevertheless, the strength of this assertion is moderate as it is based on just a few previous studies whose designs were mainly retrospective and only included a small number of selected patients (e.g., chronic obstructive pulmonary disease (COPD) was sometimes excluded) [6-8].

A prospective study was therefore conducted in a large and unselected population with the goal of clarifying whether serum CRP could identify patients with pneumonia.

\section{Patients and Methods}

2.1. Subjects. A prospective study was performed in a 500bed university hospital, and patients with the following inclusion criteria were recruited during one winter season (2013-14): (1) adults > 18 years old admitted to the emergency ward, (2) respiratory symptoms (cough, sputum production, dyspnea, tachypnea, and pleuritic pain) as the main complaint, with or without fever, and (3) disease duration of 
less than two weeks. The exclusion criteria were (1) a final diagnosis of acute decompensated heart failure, pulmonary embolism, lung cancer, or an upper respiratory infection (e.g., acute pharyngitis, rhinitis, and sinusitis), (2) severe immunosuppression (e.g., human immunodeficiency virus infection and hematological diseases) or receiving immunosuppressive therapy (i.e., prednisone or an equivalent dose of $15 \mathrm{mg}$ daily for 2 weeks or other immunosuppressant drugs), and (3) no hospitalization required.

Hospitalization was considered necessary if patients met one of the following: (1) need for either respiratory support (Sa $02<90 \%$ or Pa $02 /$ Fi $02<300$ ), mechanical ventilation (respiratory acidosis with $\mathrm{pH}<7.30$ ), or vasopressor drugs, (2) worsening of associated comorbidities (e.g., decompensated heart failure), (3) inability to take oral drugs, or (4) no response to an initial adequate treatment in the emergency department.

The local ethics committee approved this study and written informed consent was obtained from each patient.

2.2. Measurements. At the initial visit to the emergency department, demographic and basic clinical information was collected from each patient. In addition to routine blood tests, a serum sample was obtained to measure CRP. Microbiological studies included sputum sampling for Gram staining and culture in all patients with LRTI, when possible, as well as blood cultures, and Streptococcus pneumoniae and Legionella pneumophila antigen detection tests in urine samples from those with pneumonia. Serology was ordered according to the criterion of the attending physician. To stratify severity in pneumonia patients, a validated prediction rule was used, namely, the CRB65 Severity Index [9]. Antibiotic therapy was administered in the emergency department based on the clinician's judgment.

Blood samples for CRP were analyzed by a particleenhanced turbidimetric assay following the manufacturer's instructions (Beckman Coulter, USA). The range of detection for this CRP assay is from 0.2 to $480 \mathrm{mg} / \mathrm{L}$.

2.3. Disease Criteria. LRTI was defined by the presence of at least one respiratory symptom (e.g., cough, sputum production, dyspnea, tachypnea, and pleuritic pain) plus at least one finding during auscultation (i.e., crackles) or one sign of infection (temperature $>38^{\circ} \mathrm{C}$, shivering, leukocyte count $>10$, or $<4 \times 10^{9}$ cells), regardless of antibiotic use. For pneumonia, a new infiltrate on the chest radiograph was also required. COPD was defined by postbronchodilator spirometric criteria, according to the Global Initiative for Chronic Obstructive Lung Disease (GOLD) guidelines, as $\mathrm{FEV}_{1} / \mathrm{FVC}$ ratio $<70 \%$. Acute bronchitis was defined as LRTI in the absence of an underlying lung disease (COPD) or focal chest infiltrates on chest $\mathrm{X}$-rays $[10,11]$. The chest $\mathrm{X}$-rays were reviewed by two clinicians with expertise in chest infections.

The diagnosis of heart failure was made on clinical grounds (history, physical examination, chest radiograph, electrocardiogram, echocardiogram, and response to diuretic treatment), according to the American College of Cardiology/American Heart Association guidelines [12]. Pulmonary embolism was the final diagnosis when intraluminal filling defects were observed in computed tomographic pulmonary angiography.

2.4. Statistical Analysis. Results are reported as means (SD) or medians (quartiles) as appropriate. Comparisons between groups were performed with $\chi^{2}$ and Fisher's exact tests for categorical variables and the nonparametric Kruskal-Wallis and Mann-Whitney $U$ tests for continuous variables. Sensitivity, specificity, and positive and negative likelihood ratios (LR), with confidence intervals based on exact binomial distribution, were calculated using standard methods. The area under the receiver operating characteristic curve (AUC) was used to establish the optimum cut-off points for CRP and leukocyte counts. Multilevel LRs were calculated as previously described with the use of equally spaced cut-off points [13]. Statistical significance was established at $p \leq 0.05$. Calculations were performed with statistical software SPSS version 22.0 (Chicago, IL, USA).

\section{Results and Discussion}

3.1. Results. A total of 1473 consecutive patients admitted to the emergency ward with acute respiratory symptoms were initially recruited, of whom 550 were excluded because of diagnoses other than LRTI (309), immunosuppressive condition or therapy (52), or no hospitalization requirement (189). Therefore, 923 patients with the final diagnoses of pneumonia (557) or other LRTI (366), namely, acute bronchitis and acute exacerbation of COPD, were included (Table 1).

Patients with pneumonia had a median CRB65 score of 2 (IQR, 1-5) and microorganisms were found in 171 (30.7\%), as follows: Streptococcus pneumoniae (118), Haemophilus influenzae (23), Chlamydophila pneumoniae (7), Legionella pneumophila (5), influenza A (5), Pseudomonas aeruginosa (4), Mycobacterium tuberculosis (4), Mycoplasma pneumoniae (2), and one for each of Moraxella catarrhalis, Staphylococcus aureus, Escherichia coli, and Enterococcus faecium. In comparison with other LRTI, patients with pneumonia were younger and had fewer comorbid conditions, higher temperatures, and both higher blood leukocyte counts and serum CRP levels. Additionally, more patients with pneumonia required admission to the intensive care unit, although in-hospital mortality was similar between groups (Table 1).

In a logistic regression model, only 4 variables were independently related to pneumonia diagnosis: under 70 years of age (OR 2.83; 95\% CI 1.95-4.09), temperature $>38^{\circ} \mathrm{C}$ (OR 2.51, 95\% CI 1.65-3.81), leukocyte count $>15 \times 10^{9} / \mathrm{L}$ (OR 2.21, 95\% CI 1.5-3.25), and serum CRP > $150 \mathrm{mg} / \mathrm{L}$ (OR 10.44, 95\% CI 7.24-15.05).

Serum CRP levels according to disease etiologies are shown in Figure 1. Subjects with pneumonia had higher serum CRP concentrations (median $187 \mathrm{mg} / \mathrm{L}$ ) than those with exacerbations of COPD $(63 \mathrm{mg} / \mathrm{L})$ or acute bronchitis $(54 \mathrm{mg} / \mathrm{L}, p<0.01)$. The CRP reached AUC of 0.84 (95\% CI $0.82-0.87$ ) to distinguish pneumonia from other LRTI. The operating characteristics of different cut-off serum CRP values are shown in Table 2. For example, a serum CRP > $200 \mathrm{mg} / \mathrm{L}$ identified pneumonia with a sensitivity, specificity, 
TABLE 1: Baseline characteristics of patients admitted with lower respiratory tract infections.

\begin{tabular}{|c|c|c|c|}
\hline & $\begin{array}{l}\text { Pneumonia } \\
\quad(n=557)\end{array}$ & $\begin{array}{l}\text { Other lower respiratory } \\
\text { tract infections* } \\
(n=366)\end{array}$ & $p$ value \\
\hline \multicolumn{4}{|l|}{ Demographics } \\
\hline Age, years & $72(56-80)$ & $79(71-85)$ & $<0.01$ \\
\hline Gender, male & $349(63)$ & $232(54)$ & 0.84 \\
\hline Comorbidity (Charlson index) & $4(2-6)$ & $6(4-7)$ & $<0.01$ \\
\hline \multicolumn{4}{|l|}{ Clinical variables } \\
\hline Days of symptoms & $4(2-7)$ & $4(2-7)$ & 0.27 \\
\hline Previous antibiotic treatment & $164(33)$ & $100(30)$ & 0.40 \\
\hline Heart rate (bpm) & $98(84-110)$ & $97(84-110)$ & 0.56 \\
\hline Respiratory rate (rpm) & $28(24-32)$ & $28(24-32)$ & 0.79 \\
\hline Systolic blood pressure (mmHg) & $123(109-140)$ & $133(116-146)$ & $<0.01$ \\
\hline Diastolic blood pressure $(\mathrm{mmHg})$ & $69(60-78)$ & $71(63-82)$ & $<0.01$ \\
\hline \multicolumn{4}{|l|}{ Temperature $\left({ }^{\circ} \mathrm{C}\right)$} \\
\hline No fever $\left(<37^{\circ} \mathrm{C}\right)$ & $197(35)$ & $219(60)$ & \multirow{3}{*}{$<0.01$} \\
\hline Low-grade fever $\left(37-38^{\circ} \mathrm{C}\right)$ & $163(29)$ & $87(24)$ & \\
\hline High-grade fever $\left(>38^{\circ} \mathrm{C}\right)$ & $197(35)$ & $60(16)$ & \\
\hline \multicolumn{4}{|l|}{ Laboratory findings } \\
\hline Basal $\mathrm{pO}_{2}(\mathrm{mmHg})$ & $61(55-70)$ & $62(55-72)$ & 0.19 \\
\hline C-reactive protein $(\mathrm{mg} / \mathrm{L})$ & $187(123-278)$ & $59(24-108)$ & $<0.01$ \\
\hline Leukocyte count $\left(\times 10^{9} / \mathrm{L}\right)$ & $13.3(9.27-17.65)$ & $10.8(7.97-13.30)$ & $<0.01$ \\
\hline Creatinine (mg/dL) & $1.0(0.8-1.3)$ & $0.9(0.7-1.2)$ & 0.01 \\
\hline \multicolumn{4}{|l|}{ Microbiology findings } \\
\hline Microorganism found & $169(30.3 \%)$ & $30(8.9 \%)$ & $<0.01$ \\
\hline \multicolumn{4}{|l|}{ Follow-up } \\
\hline Days in hospital & $6(4-10)$ & $6(4-9)$ & 0.05 \\
\hline Intensive care unit transfers & $37(7)$ & $4(1)$ & $<0.01$ \\
\hline In-hospital mortality & $31(6)$ & $16(4)$ & 0.45 \\
\hline
\end{tabular}

Quantitative variables are shown as medians (IQR 25-75) and qualitative variables as absolute numbers (percentages). ${ }^{*}$ Other lower respiratory tract infections included acute bronchitis and acute exacerbations of COPD.

TABLE 2: Operating characteristics of C-reactive protein for identifying pneumonia according to different serum values.

\begin{tabular}{|c|c|c|c|c|}
\hline Serum CRP $(\mathrm{mg} / \mathrm{L})$ & Sensitivity, \% (95\% CI) & Specificity, \% (95\% CI) & LR+ & LR- \\
\hline$\geq 50$ & $91.3(88.7-93.4)$ & $43.9(38.9-49.1)$ & $1.6(1.4-1.7)$ & $0.2(0.1-0.2)$ \\
\hline$\geq 100$ & $82.4(79.0-85.3)$ & $72.3(67.6-76.7)$ & $3(2.5-3.5)$ & $0.2(0.2-0.2)$ \\
\hline$\geq 150$ & $65.1(61.1-69.0)$ & $87.16(83.2-90.2)$ & $5.0(3.8-6.6)$ & $0.4(0.3-0.4)$ \\
\hline$\geq 200$ & $44.8(40.8-49.0)$ & $95.6(93.0-97.2)$ & $10.2(6.3-16.6)$ & $0.5(0.5-0.6)$ \\
\hline
\end{tabular}

CRP, C-reactive protein; LR, likelihood ratio.

and positive and negative LR of $44.8 \%, 95.6 \%$, and 10.2 and 0.5 , respectively. Moreover, the positive LR for several CRP intervals was calculated (Table 3 ). Thus, CRP intervals above $200 \mathrm{mg} / \mathrm{L}$ were associated with LR positive greater than 5 (for which pneumonia is likely), whereas CRP intervals below $75 \mathrm{mg} / \mathrm{L}$ were associated with LR lower than 0.2 (for which pneumonia is unlikely).

It was also observed that CRP levels were not related to the variable "days of symptoms." Indeed, median CRP values in patients with $\leq 2,3-5$, and $\geq 6$ days of symptoms were
$107 \mathrm{mg} / \mathrm{L}$ (45-196), $127 \mathrm{mg} / \mathrm{L}$ (45-214), and $123 \mathrm{mg} / \mathrm{L}$ (43$222)$, respectively $(p=0.70)$.

3.2. Discussion. This study showed that serum CRP measurements upon admission to the hospital are useful for distinguishing patients with pneumonia from those with other LRTI.

Previous studies have investigated the utility of serum CRP in identifying pneumonia. In a retrospective analysis of 60 patients with LRTI, 75\% of patients with pneumonia 
TABLE 3: Multilevel likelihood ratios for different serum C-reactive protein intervals.

\begin{tabular}{lccc}
\hline $\begin{array}{l}\text { Serum CRP } \\
(\mathrm{mg} / \mathrm{L})\end{array}$ & $\begin{array}{c}\text { Pneumonia } \\
(n=557)\end{array}$ & $\begin{array}{c}\text { Other lower } \\
\text { respiratory tract } \\
\text { infections } \\
(n=366)\end{array}$ & LR+ \\
\hline$>250$ & 171 & 7 & $16.3(7.7-34.4)$ \\
$225-250$ & 40 & 5 & $5.3(2.1-13.4)$ \\
$200-225$ & 32 & 4 & $5.3(1.9-15.0)$ \\
$175-200$ & 53 & 17 & $2.0(1.2-3.5)$ \\
$150-175$ & 58 & 13 & $2.9(1.6-5.3)$ \\
$125-150$ & 46 & 23 & $1.3(0.8-2.1)$ \\
$100-125$ & 49 & 30 & $1.1(0.7-1.6)$ \\
$75-100$ & 31 & 42 & $0.5(0.3-0.7)$ \\
$50-75$ & 19 & 60 & $0.2(0.1-0.3)$ \\
$25-50$ & 27 & 67 & $0.2(0.1-0.4)$ \\
$<25$ & 20 & 98 & $0.1(0.0-0.2)$ \\
\hline
\end{tabular}

CRP, C-reactive protein; LR, likelihood ratio.

* Other lower respiratory tract infections included acute bronchitis and acute exacerbations of COPD.

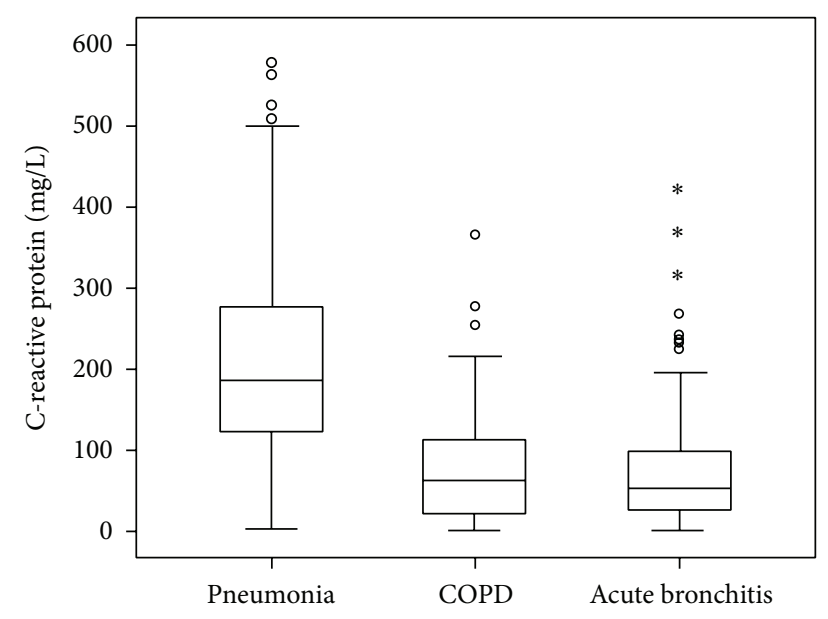

COPD: chronic obstructive pulmonary disease

FIGURE 1: C-reactive protein levels in the study population. * represents extreme values.

had serum CRP levels $>100 \mathrm{mg} / \mathrm{L}$, although no information was reported on specificity [6]. In a prospective study of 97 patients with pneumonia, it was found that only $5 \%$ had serum CRP levels below $50 \mathrm{mg} / \mathrm{L}$ [7]. In another prospective study of 284 patients with LRTI, a serum CRP $>100 \mathrm{mg} / \mathrm{L}$ had a specificity of $96 \%$ for labeling pneumonia [8], even though patients with acute exacerbation of COPD were excluded from the analysis. Based on these studies, the British Thoracic Society stated that the measurement of serum CRP on admission may be helpful in distinguishing pneumonia from other LRTI, with moderate weight being placed on this recommendation [4].
More recently, in a post hoc analysis of 545 patients with LRTI, Müller et al. [14] found that serum CRP had AUC of 0.76 in identifying patients with pneumonia. However, only $11 \%$ of patients included had acute exacerbation of COPD, and the technique used (a highly sensitive CRP) is not widely available in clinical practice. Finally, Bafadhel et al. [15] studied 158 patients with LRTI and concluded that a cut-off point for serum CRP of $>48 \mathrm{mg} / \mathrm{L}$ had a sensitivity and specificity of $91 \%$ and $93 \%$ for pneumonia, respectively. Even so, patients with acute exacerbations of COPD were also excluded from this analysis.

Previous studies usually recommended a single CRP cutoff point to dichotomize respiratory infections into either pneumonia or nonpneumonia categories. This approach eliminates much of the diagnostic information contained in laboratory tests that have continuous integer values. An alternative strategy for improving the discriminative properties of diagnostic tests is to generate multilevel LRs using various cut-off points and then apply them to convert the pretest probabilities into posttest probabilities of having pneumonia [16]. This new strategy, when applied to laboratory results in the borderline pneumonia range with the use of single cut-off points, generates low LRs that will not misclassify patients if the pretest suspicion for pneumonia is low. Medical literature commonly describes the operating characteristics of a diagnostic test by dichotomizing test results into normal and abnormal values and calculating their sensitivity and specificity. Unfortunately, this knowledge offers little clinical utility when evaluating individual patients because these indexes do not describe the probability of disease if the result is positive or negative, as LR does. Moreover, the rationale behind the use of multilevel LRs is that the dichotomization of test results does not assist in assessing to what degree a test result alters a clinician's estimation of the pretest probability of disease [13].

In our study, we sought to improve upon the shortcomings of the previous ones. First, this is the largest study performed on this issue to date. Second, the population was derived from unselected patients admitted to the emergency department with acute respiratory symptoms, including those with acute exacerbations of COPD. Third, the design of the study was prospective. Finally, the multilevel LRs for several intervals of serum CRP provided useful information on the posttest probability of having pneumonia. Thus, serum CRP intervals above $200 \mathrm{mg} / \mathrm{L}$ were associated with LR positive $>5$ (for which pneumonia is likely), whereas CRP intervals below $75 \mathrm{mg} / \mathrm{L}$ were associated with $\mathrm{LR}<0.2$ (for which pneumonia is unlikely). Between these intervals, the serum CRP did not provide useful clinical information.

One drawback of the study was that detailed information on physical signs was not provided. However, previous studies have shown that there are no findings from the history or physical examination capable of confidently ruling in or out the diagnosis of pneumonia [2]. Also, other potentially useful biomarkers of infection were not tested. For instance, procalcitonin has been reported to have high discriminative power in identifying pneumonia [17] although it is not widely available in emergency settings. 


\section{Conclusion}

The results of our study suggest that the routine use of serum CRP levels in hospitalized patients with acute respiratory symptoms can help clinicians to differentiate pneumonia from other respiratory infections. Indeed, serum CRP levels above $200 \mathrm{mg} / \mathrm{L}$ or below $75 \mathrm{mg} / \mathrm{L}$ make the diagnosis of pneumonia likely or unlikely, respectively. A further prospective validation of CRP ranges in an independent population is warranted.

\section{Competing Interests}

The authors state that they have no competing interests including any financial, personal, or other relationships with other people or organizations with the submitted work that could inappropriately influence or be perceived to influence their work.

\section{References}

[1] S. Jain, W. H. Self, and R. G. Wunderink, "Community-acquired pneumonia requiring hospitalization," The New England Journal of Medicine, vol. 373, no. 24, pp. 415-427, 2015.

[2] J. P. Metlay, W. N. Kapoor, and M. J. Fine, "Does this patient have community-acquired pneumonia? Diagnosing pneumonia by history and physical examination," The Journal of the American Medical Association, vol. 278, no. 17, pp. 1440-1445, 1997.

[3] E. E. Walsh, A. J. Swinburne, K. L. Becker et al., "Can serum procalcitonin levels help interpret indeterminate chest radiographs in patients hospitalized with acute respiratory illness?" Journal of Hospital Medicine, vol. 8, no. 2, pp. 61-67, 2013.

[4] W. S. Lim, S. V. Baudouin, R. C. George et al., "British Thoracic Society guidelines for the management of community acquired pneumonia in adults: update 2009," Thorax, vol. 64, supplement 3, pp. iiil-iii55, 2009.

[5] F. Blasi, M. Bocchino, F. Di Marco, L. Richeldi, and S. Aliberti, "The role of biomarkers in low respiratory tract infections," European Journal of Internal Medicine, vol. 23, no. 5, pp. 429435, 2012.

[6] R. P. Smith, B. J. Lipworth, I. A. Cree, E. M. Spiers, and J. H. Winter, "C-reactive protein: a clinical marker in communityacquired pneumonia," Chest, vol. 108, no. 5, pp. 1288-1291, 1995.

[7] L.-O. Hansson, J. U. Hedlund, and A. B. Ortqvist, "Sequential changes of inflammatory and nutritional markers in patients with community-acquired pneumonia," Scandinavian Journal of Clinical and Laboratory Investigation, vol. 57, no. 2, pp. 111118, 1997.

[8] A. Castro-Guardiola, A. Armengou-Arxé, A.-L. ViejoRodríguez, G. Peñarroja-Matutano, and F. Garcia-Bragado, "Differential diagnosis between community-acquired pneumonia and non-pneumonia diseases of the chest in the emergency ward," European Journal of Internal Medicine, vol. 11, no. 6, pp. 334-339, 2000.

[9] J. D. Chalmers, A. Singanayagam, A. R. Akram et al., "Severity assessment tools for predicting mortality in hospitalised patients with community-acquired pneumonia. Systematic review and meta-analysis," Thorax, vol. 65, no. 10, pp. 878-883, 2010.

[10] Global Initiative for Chronic Obstructive Lung Disease, Global Strategy for the Diagnosis, Management, and Prevention of
Chronic Obstructive Pulmonary Disease, Global Initiative for Chronic Obstructive Lung Disease, 2015, http://www.goldcopd .it/materiale/2015/GOLD_Report_2015.pdf.

[11] M. Woodhead, F. Blasi, S. Ewig et al., "Joint Taskforce of the European Respiratory Society and European Society for Clinical Microbiology and Infectious Diseases. Guidelines for the management of adult lower respiratory tract infections," Clinical Microbiology and Infection, vol. 17, supplement 6, pp. ele59, 2011.

[12] C. W. Yancy, M. Jessup, B. J. Bozkurt et al., "2013 ACCF/AHA Guideline for the management of heart failure. A report of the American College of Cardiology Foundation/American Heart Association Task Force on Practice Guidelines," Circulation, vol. 128, no. 16, pp. e240-e327, 2013.

[13] R. Jaeschke, G. Guyatt, and D. Sackett, "User's guides to the medical literature: III: how to use an article about a diagnostic test," Journal of the American Medical Association, vol. 271, pp. 703-707, 1994.

[14] B. Müller, S. Harbarth, D. Stolz et al., "Diagnostic and prognostic accuracy of clinical and laboratory parameters in community-acquired pneumonia," BMC Infectious Diseases, vol. 7, article 10, 2007.

[15] M. Bafadhel, T. W. Clark, C. Reid et al., "Procalcitonin and Creactive protein in hospitalized adult patients with communityacquired pneumonia or exacerbation of asthma or COPD," Chest, vol. 139, no. 6, pp. 1410-1418, 2011.

[16] J. E. Heffner, S. A. Sahn, and L. K. Brown, "Multilevel likelihood ratios for identifying exudative pleural effusions," Chest, vol. 121, no. 6, pp. 1916-1920, 2002.

[17] G. A. Alba, Q. A. Truong, H. K. Gaggin et al., "Diagnostic and prognostic utility of procalcitonin in patients presenting to the emergency department with dyspnea," American Journal of Medicine, vol. 129, pp. 96-110, 2016. 


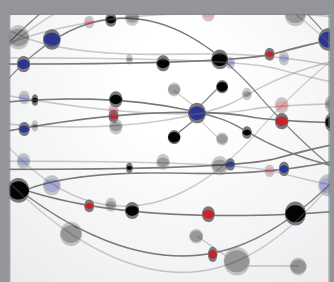

The Scientific World Journal
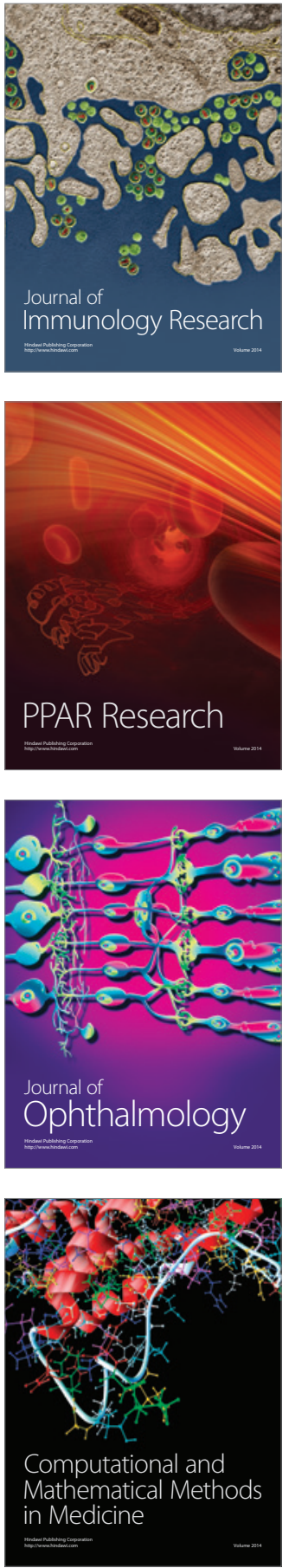

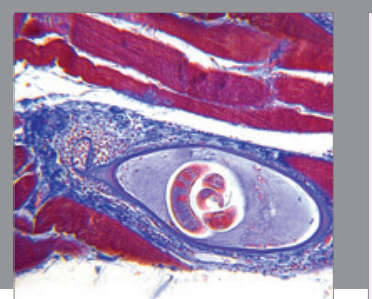

Gastroenterology Research and Practice

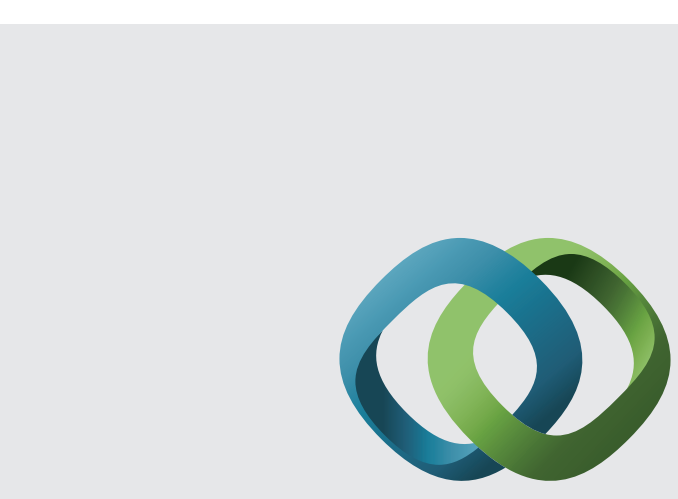

\section{Hindawi}

Submit your manuscripts at

http://www.hindawi.com
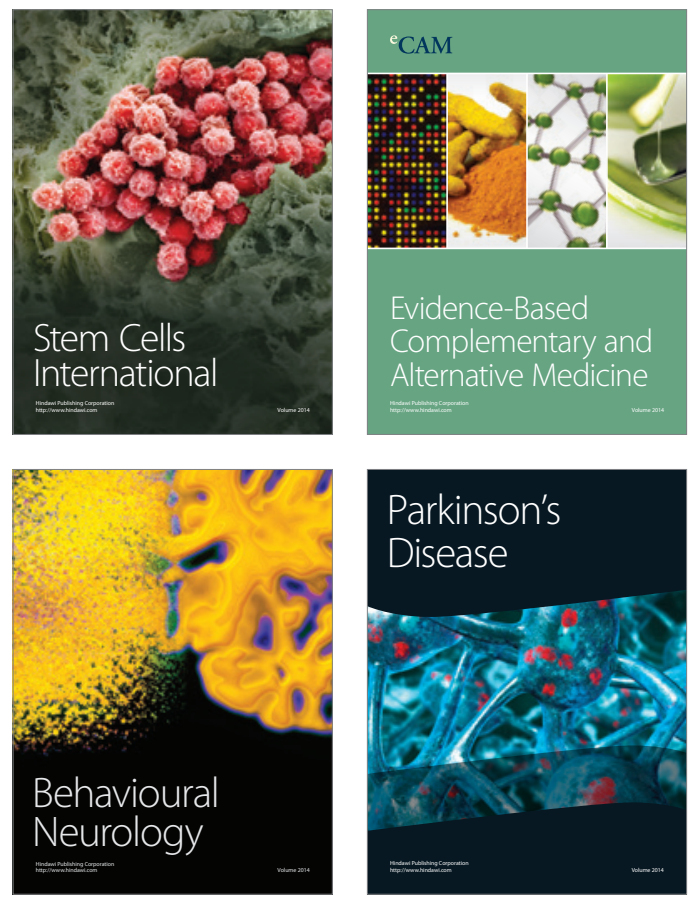
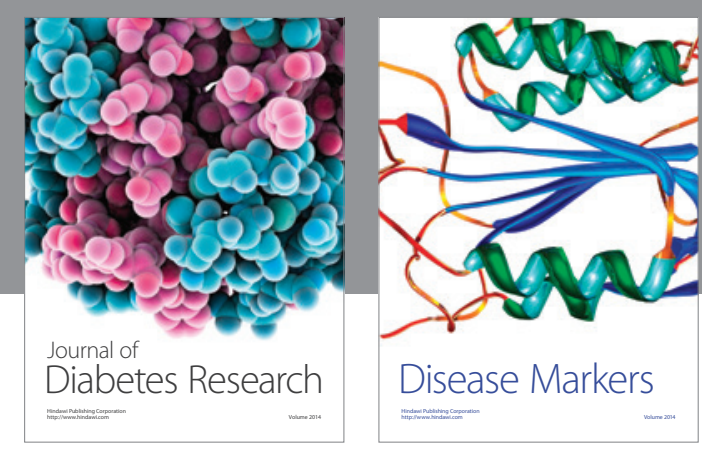

Disease Markers
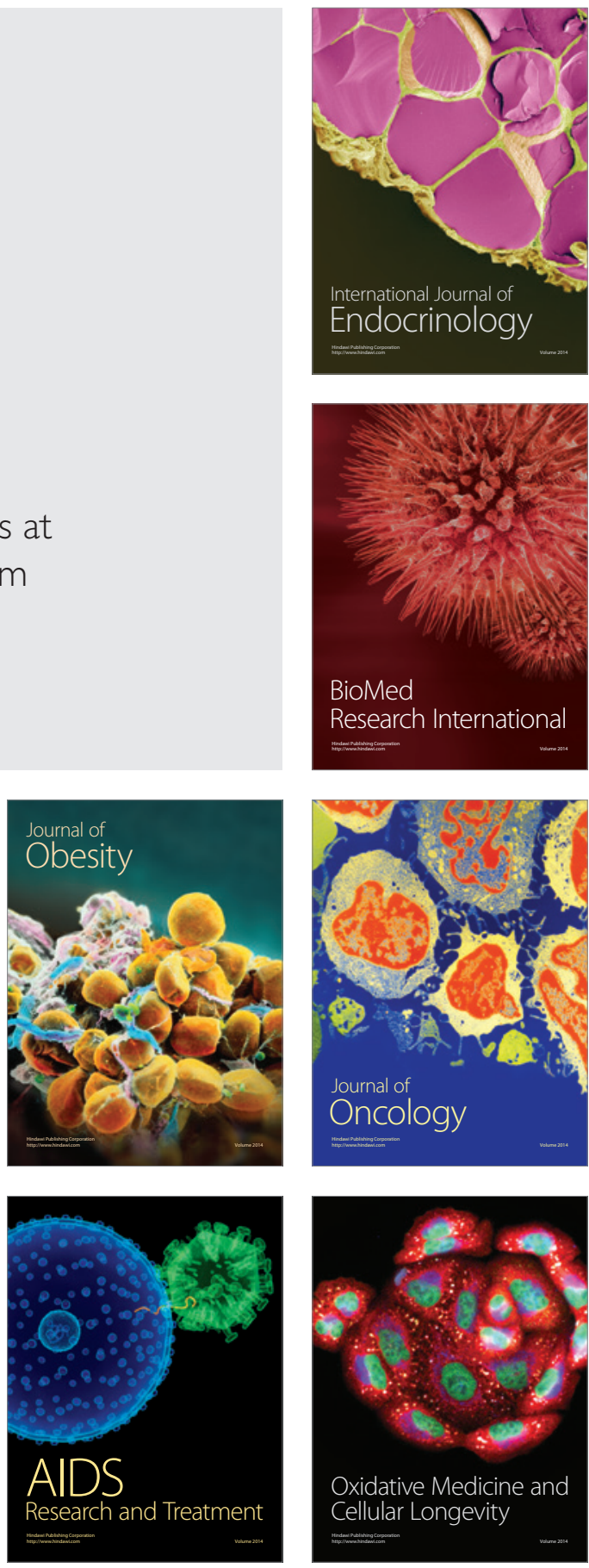